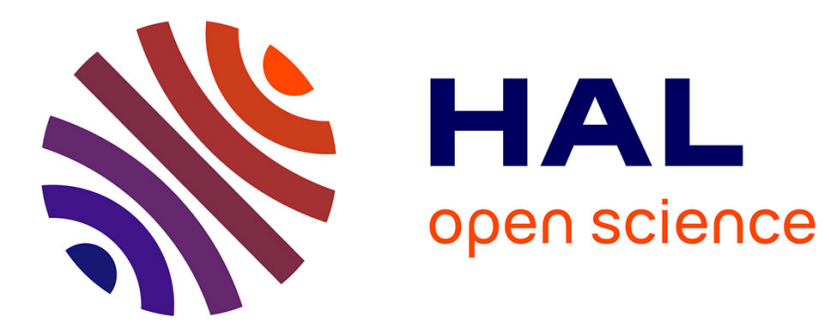

\title{
Imagerie acoustique et modélisation dans les sédiments
}

\author{
J. Bresson, R. Barriol
}

\section{To cite this version:}

J. Bresson, R. Barriol. Imagerie acoustique et modélisation dans les sédiments. Revue de Physique Appliquée, 1989, 24 (10), pp.1007-1017. 10.1051/rphysap:0198900240100100700 . jpa-00246133

\section{HAL Id: jpa-00246133 https://hal.science/jpa-00246133}

Submitted on 1 Jan 1989

HAL is a multi-disciplinary open access archive for the deposit and dissemination of scientific research documents, whether they are published or not. The documents may come from teaching and research institutions in France or abroad, or from public or private research centers.
L'archive ouverte pluridisciplinaire HAL, est destinée au dépôt et à la diffusion de documents scientifiques de niveau recherche, publiés ou non, émanant des établissements d'enseignement et de recherche français ou étrangers, des laboratoires publics ou privés. 
Classification

Physics Abstracts

$43.30-43.60-43.85-43.88$

\title{
Imagerie acoustique et modélisation dans les sédiments
}

\author{
J. Bresson et R. Barriol \\ LMAI, Université, ave. de Villeneuve, 66025 Perpignan, France \\ (Reçu le 22 décembre 1988, révisé le 2 mai 1989, accepté le 11 juillet 1989)
}

\begin{abstract}
Résumé. - Une antenne acoustique multi-éléments avec focalisation à l'émission et à la réception a été étudiée. Les résultats obtenus par modélisation avec et sans passage d'une interface sont présentés et comparés à ceux provenant des essais en bassin. Le pouvoir discriminatoire du dispositif de reconstruction d'images est défini tant théoriquement qu'expérimentalement.
\end{abstract}

\begin{abstract}
We have presented in this paper an acoustic array antenna which uses focalization for transmission and reception. Results obtained with the model (with and without interface) are compared with in situ underwater experiments. The imaging resolution is obtained both theoretically and experimentally.
\end{abstract}

\section{Introduction.}

L'intérêt croissant accordé à l'exploitation des fonds marins nécessite des moyens de reconnaissance de stratifications et d'objets enfouis dans les sédiments marins pour des applications aussi diverses que l'enrochement des câbles sous-marins, la recherche de nodules, la vision en eau turbide, etc.

Dans un domaine similaire, nous rappellerons l'étude de Shibata [1] en 1978 qui, à la fréquence de $100 \mathrm{kHz}$ et à l'aide d'un réseau circulaire d'hydrophones en réception, restitue l'image d'une cible située à $3 \mathrm{~m}$. Plus récemment, le laboratoire LMA CNRS de Marseille [2] réalisa l'image d'objets positionnés à $10 \mathrm{~cm}$ en utilisant une barette de transducteurs fonctionnant à $80 \mathrm{kHz}$.

Nous présentons l'étude théorique et expérimen-

devant opérer près de l'interface eau-sédiment et permettant de reproduire à partir du champ acoustique rétrodiffusé l'image d'objets enfouis. Le choix de la fréquence de travail de $46 \mathrm{kHz}$ résulte d'un compromis entre la pénétration de l'onde acoustique et la résolution recherchée. Ce système fonctionne en échographie séquentielle par illumination sélective de l'objet. Une partie de l'objet est éclairée à chaque «tir »; il devient nécessaire de focaliser à l'émission et à la réception afin d'assurer une meilleure détection [3, 4].

\section{Analyse du problème.}

L'antenne comporte un ensemble de 56 transducteurs acoustiques ponctuels, équirépartis sur 4 couronnes de rayons respectifs $0,125,0,18,0,3$ et $0,5 \mathrm{~m}$ de manière à former une antenne plane (Fig. 1). La dimension maximum résulte d'un compromis entre un encombrement prohibitif de l'antenne et des dimensions faibles du faisceau acoustique. D'autre part, l'importance des lobes secondaires est tributaire de la position des couronnes intermédiaires. La focalisation est assurée par l'introduction de retards et de déphasages entre les signaux des différentes couronnes [5], ce qui permet l'obtention d'une tache focale de section quasi circulaire.

- La modélisation du champ acoustique dans le cas d'un seul milieu renseigne sur les caractéristiques acoustiques e antenne . ans e cas u passage de l'interface eau-sédiment, le même algorithme est utilisé en faisant toutefois intervenir les paramètres liés au changement de milieux.

- Des essais in situ d'imagerie acoustique viennent compléter cette étude.

\section{Distribution du champ acoustique de l'antenne dans l'eau.}

La focalisation du faisceau acoustique confère à l'ensemble une directivité importante. La minimisa- 


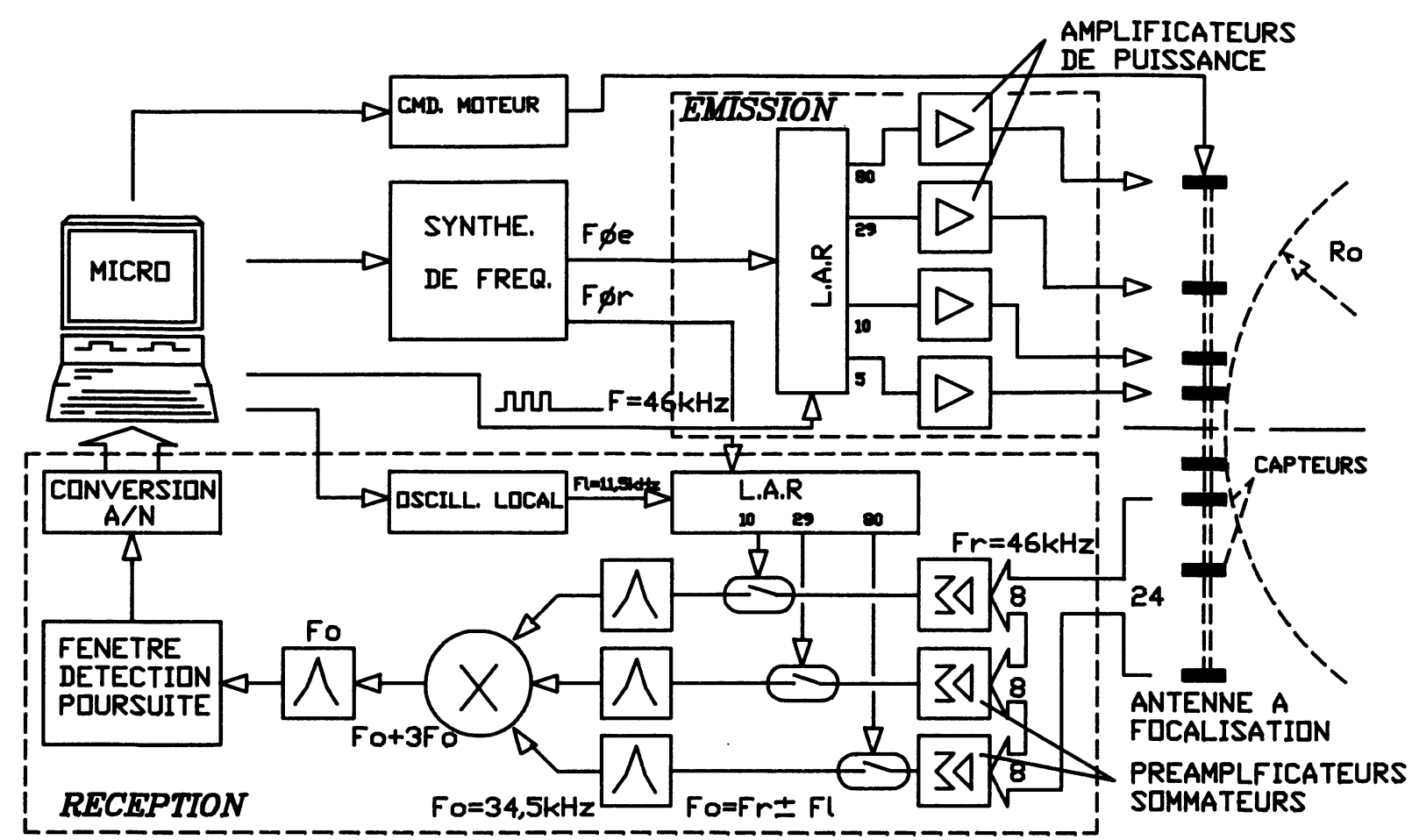

Fig. 1. - Synoptique de l'antenne à focalisation multi-éléments.

[Block diagram of the multi-elements focusing array.]

tion des lobes secondaires ou apodisation est partiellement résolue :

- en émission en optimisant les positions relatives des couronnes, l'optimisation s'effectue numériquement en faisant varier la position des couronnes médianes jusqu'à obtenir le niveau le plus faible des lobes secondaires, signal.

- en réception par un traitement particulier du

3.1 EN ÉMISSION. - Dans le cas d'ondes sphériques, la pression au point cible est la somme des pressions élémentaires provenant des 32 émetteurs omnidirectionnels équirépartis sur les 4 couronnes, soit :

$$
P_{\Sigma}=\sum_{c=1}^{4} \sum_{i=1}^{8} X_{i} \cos \left(\omega t+\phi_{i}\right)
$$

où :

$c=$ indice des couronnes $(1 \rightarrow 4)$,

$i=$ indice des capteurs $(1 \rightarrow 8)$,

$X_{i}=\left(A^{2}+B^{2}\right)^{1 / 2}, \phi_{i}=\operatorname{arctg}(B / A)$,

$A=\left(P_{0} r_{0} / r_{i}\right) \cos \left(k_{\mathrm{c}} r_{i}-\alpha_{c \mathrm{e}}\right)$,

$B=\left(P_{0} r_{0} / r_{i}\right) \sin \left(k_{\mathrm{e}} r_{i}-\alpha_{\mathrm{ce}}\right)$,

$P_{0}=$ pression émise par un capteur élémentaire à la distance $r_{0}$,

$r_{i}=$ distance entre le capteur $i$ et le point cible,

$k_{\mathrm{c}}=2 \pi F / C_{\mathrm{e}}=\omega / C_{\mathrm{e}}$ nombre d'onde dans l'eau de célérité $C_{\mathrm{e}}$ où $F$ est la fréquence de fonctionnement,

$\alpha_{c e}=$ déphasage de la couronne $c$ en émission.
Les retards introduits à l'excitation de chaque couronne ou « loi de phases » ne correspondent pas exactement à la différence de trajet de l'onde acoustique entre l'antenne plane et la concavité souhaitée, mais s'obtiennent par optimisation numérique de la réponse acoustique au point de focalisation considéré. Cet ajustement prend ainsi en compte la divergence des ondes sphériques.

L'exploitation du modèle numérique restitue la topographie en 3 dimensions du champ acoustique (Fig. 2) pour une distance de focalisation de $2 \mathrm{~m}$. Le niveau moyen des lobes secondaires est inférieur d'environ $10 \mathrm{~dB}$ à celui du lobe principal. On observe un demi-angle d'ouverture de $1,22^{\circ}$, et la profondeur de champ, définie comme la longueur de la tache focale à $-3 \mathrm{~dB}$ est de $0,75 \mathrm{~m}$.

3.2 EN RÉCEPTION. - L'approche classique sur une antenne réseau consiste à sommer les différents signaux issus des capteurs ce qui s'apparente à une transformée de Fourier discrète (TFD). Puis la pondération d'amplitude (fenêtre de pondération) permet la suppression des lobes secondaires. Un traitement semblable est appliqué en réception, où après sommation des signaux d'une même couronne, le traitement multiplicatif entre les signaux issus des trois couronnes assure la suppression quasi complète des lobes secondaires. La recherche par calculateur de la position de la couronne médiane assure ainsi l'apodisation optimum. Au niveau d'une couronne, 


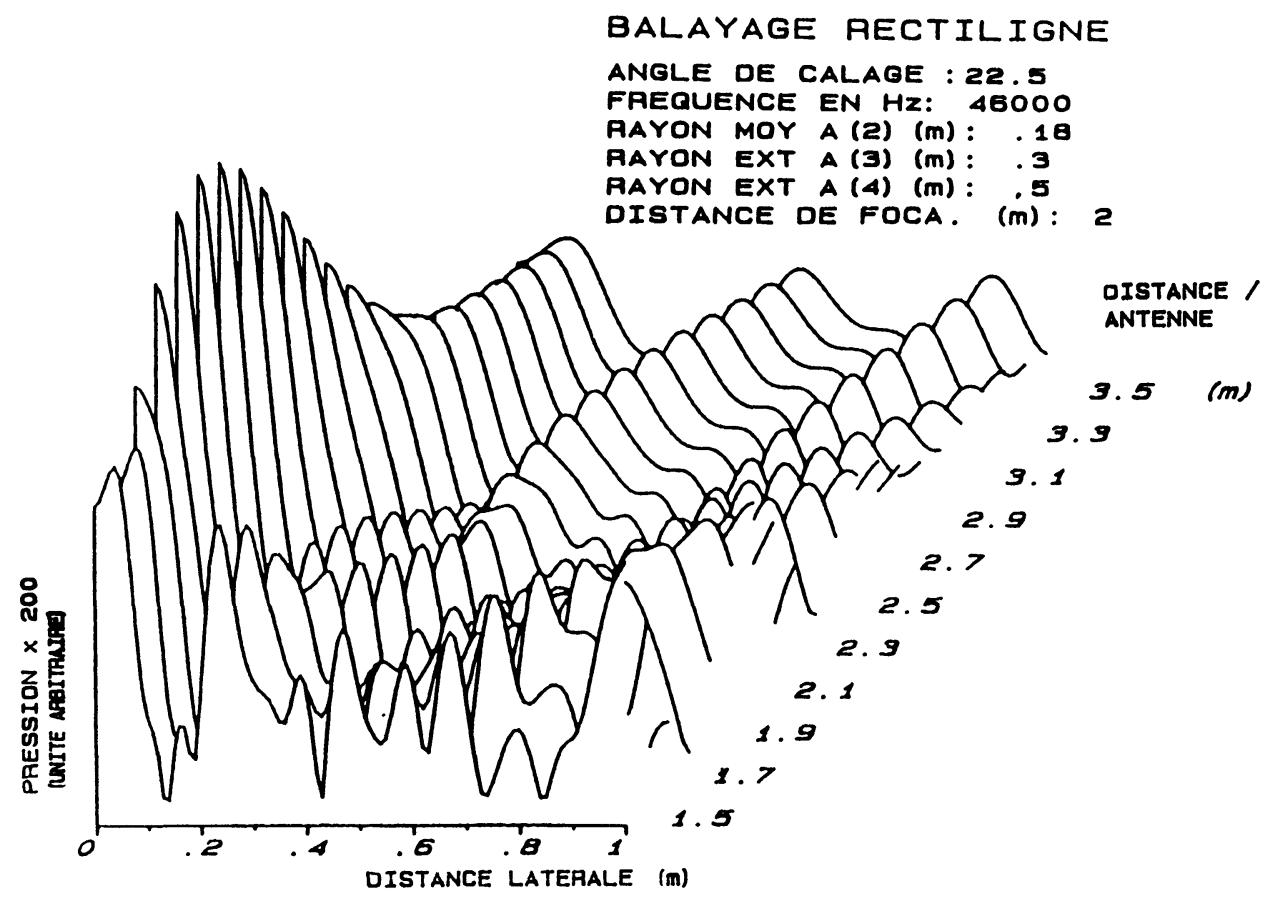

Fig. 2. - Champ acoustique théorique de l'antenne à focalisation dans l'eau (émission).

[Theorical acoustic transmission field of the focusing antenna in water.]

le signal correspondant à la somme des pressions s'écrit:

$$
P_{c}=\sum_{i=1}^{8} P_{i \mathrm{r}}=X_{c} \cos \left(\omega t+\Gamma_{c}\right)
$$

où :

$P_{i \text { r }}=$ pression élémentaire reçue par un capteur,

$X_{c}=\left(A_{c}^{2}+B_{c}^{2}\right)^{1 / 2}$,

$\Gamma_{c}=\operatorname{arctg}\left(B_{c} / A_{c}\right)$,

$A_{c}=\sum_{i=1}^{8}\left(P_{0} r_{0} / r_{i}\right) \cos k_{\mathrm{e}} r_{i}$,

$B_{c}=\sum_{i=1}^{8}\left(P_{0} r_{0} / r_{i}\right) \sin k_{\mathrm{e}} r_{i}$.

Après focalisation sur l'axe de l'antenne par introduction du déphasage $\alpha_{c r}$ au niveau de la couronne, il vient :

$$
P_{c}^{\prime}=X_{c} \cos \left(\omega t+\Gamma_{c}+\alpha_{c r}\right)=X_{c} \cos \left(\omega t+\beta_{c}\right) .
$$

Le traitement multiplicatif restitue un signal composite avec comme porteuse l'harmonique 3 modulé par le fondamental

$$
\begin{aligned}
P \pi= & \prod_{c=2}^{4} P_{c}^{\prime}= \\
= & (1 / 4)\left(\prod_{c=2}^{4} X_{c}\right) \cos \left(3 \omega t+\beta_{2}+\beta_{3}+\beta_{4}\right)+ \\
& +1 / 4\left(\prod_{c=2}^{4} X_{c}\right) Y_{c} \cos \left(\omega t+\theta_{c}\right)
\end{aligned}
$$

où :

$Y_{c}=\left(Y_{1}^{2}+Y_{2}^{2}\right)^{1 / 2}, \theta_{c}=\operatorname{arctg}\left(Y_{2} / Y_{1}\right)$,

$Y_{1}=\cos \left(\beta_{2}+\beta_{3}-\beta_{4}\right)+\cos \left(\beta_{2}+\beta_{4}-\beta_{3}\right)$

$+\cos \left(\beta_{4}+\beta_{3}-\beta_{2}\right)$,

$Y_{2}=\sin \left(\beta_{2}+\beta_{3}-\beta_{4}\right)+\sin \left(\beta_{2}+\beta_{4}-\beta_{3}\right)$

$+\sin \left(\beta_{4}+\beta_{3}-\beta_{2}\right)$.

Un filtre passe-bande centré sur le fondamental élimine l'harmonique 3 . Ainsi le fondamental, après détection crête et conversion numérique, participe à la reconstruction de l'image de l'objet.

L'exploitation de cette information aboutit à la cartographie en pression (Fig. 3). Les lobes secondaires ont disparu au voisinage du pic central, les dimensions de la tache focale à $2 \mathrm{~m}$ sont plus faibles (demi-angle d'ouverture $=0,5^{\circ}$, profondeur de champ à $-3 \mathrm{~dB}=0,36 \mathrm{~m})$.

3.3 RÉPONSE ACOUSTIQUE DE L'ANTENNE EN ÉMISSION-RÉCEPTION. - $\mathrm{La}$ réponse acoustique du reseau e trans ucteurs correspon a ors au produit des deux réponses acoustiques précédentes. Les résultats théoriques (Fig. 4) laissent espérer, à $2 \mathrm{~m}$ de distance, une tache focale de dimensions réduites (angle d'ouverture à $-3 \mathrm{~dB}=1^{\circ}$, longueur de la tache focale à $-3 \mathrm{~dB}=0,31 \mathrm{~m}$ ) avec des niveaux relatifs des lobes secondaires de $-25 \mathrm{~dB}$.

\section{Traversée d'une interface.}

Nous considérerons ici le cas de 2 milieux homogènes isotropes de caractéristiques mécaniques, acousti- 


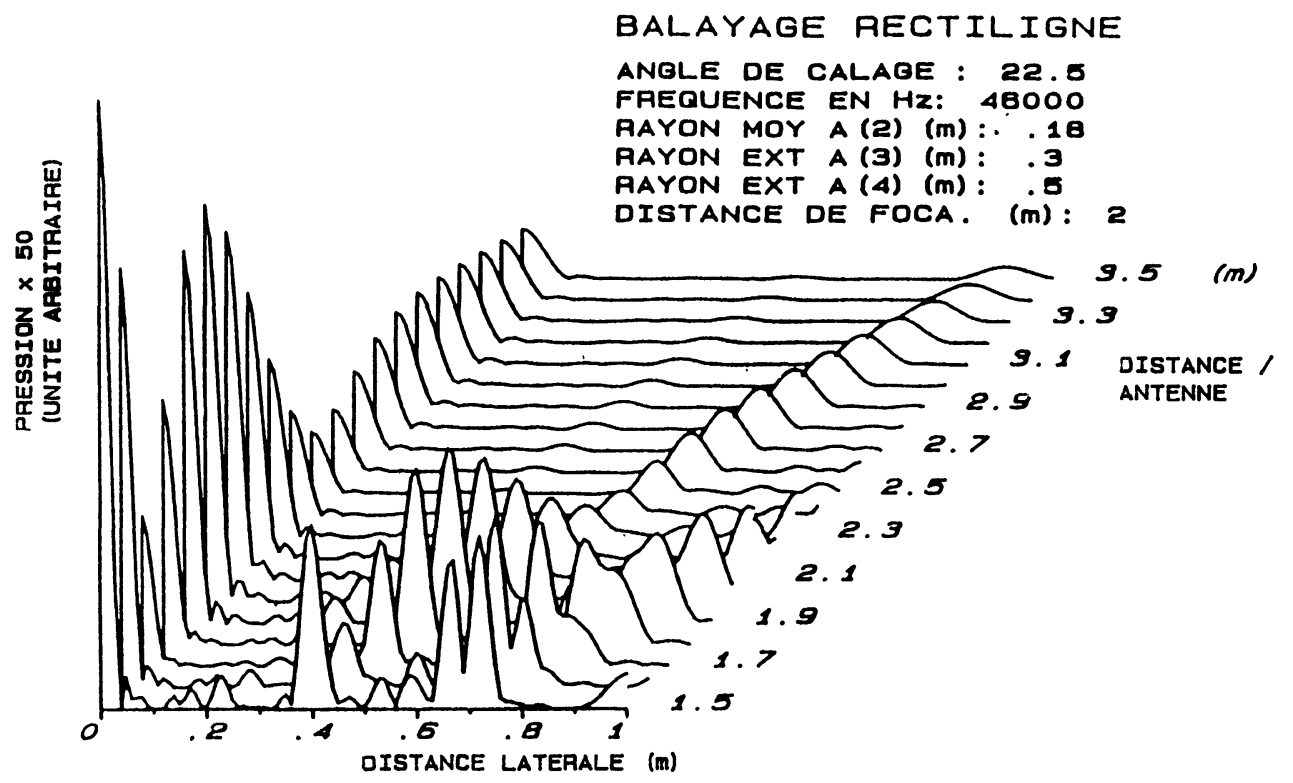

Fig. 3. - Champ acoustique théorique de l'antenne à focalisation dans l'eau (réception).

[Theorical acoustic reception field of the focusing antenna in water.]

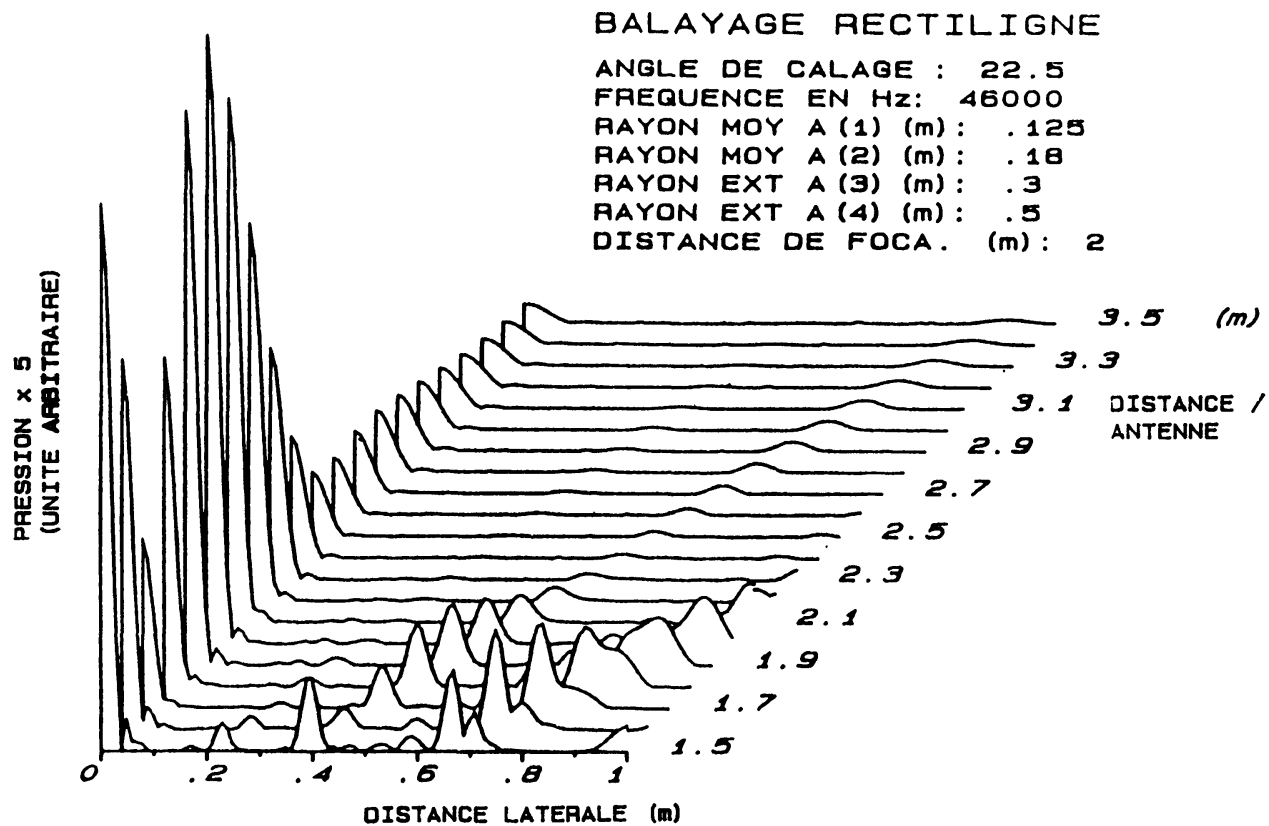

Fig. 4. - Champ acoustique théorique de l'antenne à focalisation dans l'eau (émission-réception).

[Theorical acoustic transmission-reception field of the focusing antenna in water.]

ques et viscoélastiques connues (masse volumique $\rho$, célérité longitudinale $C$, atténuation de l'onde acoustique $\alpha$ ). D'autre part, seul le comportement $\mathrm{du}$ faisceau acoustique réfracté sera pris en compte. La traversée d'une interface s'accompagne alors :

- d'une réfraction d'angle $\alpha_{1}$ du faisceau acoustique incident, d'angle $\alpha_{2}$,
- d'une perte d'énergie liée au changement de milieux avec,

$$
T=1-r^{2}=1-\left[\frac{Z_{\mathrm{s}} \cos \alpha_{1}-Z_{\mathrm{e}} \cos \alpha_{2}}{Z_{\mathrm{s}} \cos \alpha_{1}+Z_{\mathrm{e}} \cos \alpha_{2}}\right]^{2}
$$

où $Z_{\mathrm{s}}=\rho_{\mathrm{s}} C_{\mathrm{s}}$ et $Z_{\mathrm{e}}=\rho_{\mathrm{e}} C_{\mathrm{e}}$ sont les impédances acoustiques du sédiment et de l'eau, 
- d'une absorption de l'énergie acoustique par le sédiment dépendant de la nature du sédiment et de la fréquence de l'onde acoustique selon une loi de la forme $\alpha(\mathrm{dB} / \mathrm{m})=K_{\mathrm{s}} F^{n}$ où la fréquence $F$ est exprimée en $\mathrm{kHz}$ et le coefficient $n$ dépend du sédiment considéré [8].

Ainsi, la pression élémentaire reçue en un point sous l'interface eau-sédiment s'écrit :

$$
\begin{aligned}
P_{\text {sed }}=\left(P_{0} r_{0} /\left(N r_{1}+\right.\right. & \left.\left.r_{2}\right)\right)(1+r) \times \\
& \times \cos \left(\omega t-k_{\mathrm{e}} r_{1}+k_{\mathrm{s}} r_{2}\right) \mathrm{e}^{-\delta_{s} r_{2}}
\end{aligned}
$$

où :

$k_{\mathrm{c}}$ et $k_{\mathrm{s}}$ sont les nombres d'onde dans l'eau et le sédiment,

$r_{1}$ et $r_{2}$ sont les trajets acoustiques dans l'eau et le sédiment,

$\delta_{\mathrm{s}}=\alpha / 8,68$ est le coefficient d'atténuation en pression du sédiment.

$N=C_{\mathrm{c}} \cos \alpha_{1} / C_{\mathrm{s}} \cos \alpha_{2}$ fait intervenir le phénomène d'aberration de la tache focale lié au fait qu'après passage de l'interface eau-sédiment la courbure de l'onde réfractée semble provenir du point image du point source.

En utilisant le même algorithme de modélisation que précédemment, nous obtenons, pour la distance de focalisation $2 \mathrm{~m}$ et pour plusieurs angles d'inclinaison de l'antenne, des cartes isobares du champ acoustique sous l'interface (Figs. 5, 6, 7 avec $C_{\mathrm{c}}=1500 \mathrm{~m} / \mathrm{s}, \quad C_{\mathrm{s}}=1600 \mathrm{~m} / \mathrm{s}, \quad \rho_{\mathrm{e}}=1000 \mathrm{~kg} / \mathrm{m}^{3}$, $\left.\rho_{\mathrm{s}}=1800 \mathrm{~kg} / \mathrm{m}^{3}, K_{\mathrm{s}}=0,01 \mathrm{~dB} / \mathrm{m} / \mathrm{kHz}\right)$.
Exploitation DU MODÈle. - Dans le cas de la traversée d'une interface, nous constatons que :

- la loi de Snell-Descartes est respectée entre le faisceau focalisé incident et réfracté ;

- les niveaux des lobes secondaires autour de la tache focale sont très faibles, inférieurs à $-30 \mathrm{~dB}$;

- les lobes secondaires de niveaux importants près de l'interface eau-sédiment seront rejetés par un filtrage temporel asservi à la distance de focalisation.

L'exploitation plus approfondie de ce modèle, notamment en considérant l'influence des paramètres géoacoustiques du sédiment, amène les conclusions suivantes en accord avec certains auteurs [9] :

- l'augmentation de la célérité dans le sédiment, pour un angle d'incidence fixe, entraîne une diminution de la longueur de la tache de focalisation qui corrélativement se rapproche de l'interface ;

- l'inclinaison de l'antenne s'accompagne d'une légère défocalisation au point initial avec un rapprochement du maximum de pression en fonction de l'accroissement de l'angle d'incidence ;

- le caractère viscoélastique du sédiment induit un important affaissement du maximum de pression au point focal.

La largeur de la tache focale ne semble pas affectée par l'évolution des paramètres géoacoustiques.

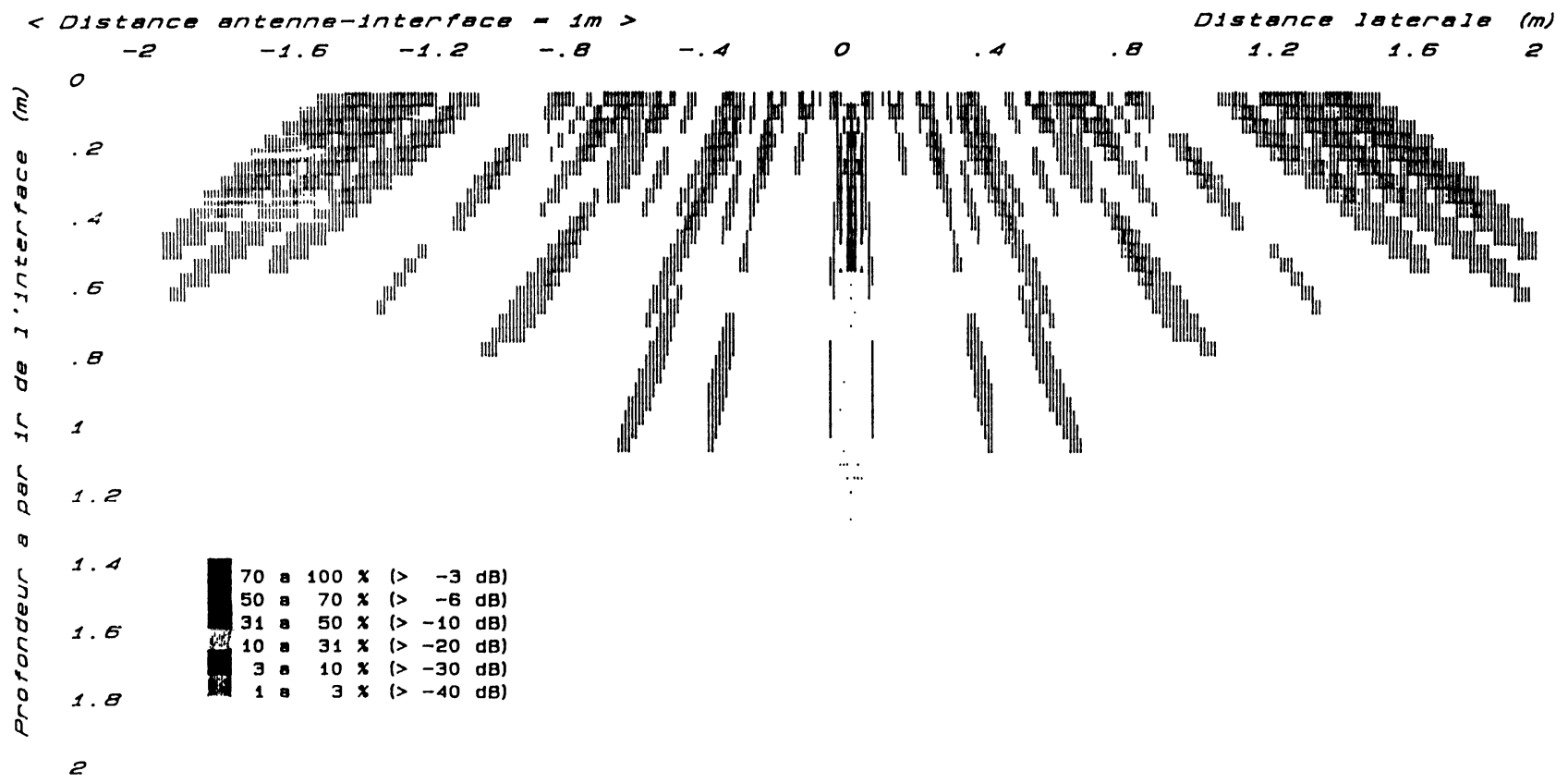

Fig. 5. - Modélisation du champ acoustique sous l'interface eau-sédiment en émission-réception (inclinaison $=0$ degré).

[Transmission-reception acoustic field model through an interface (inclination $=0$ degree).] 


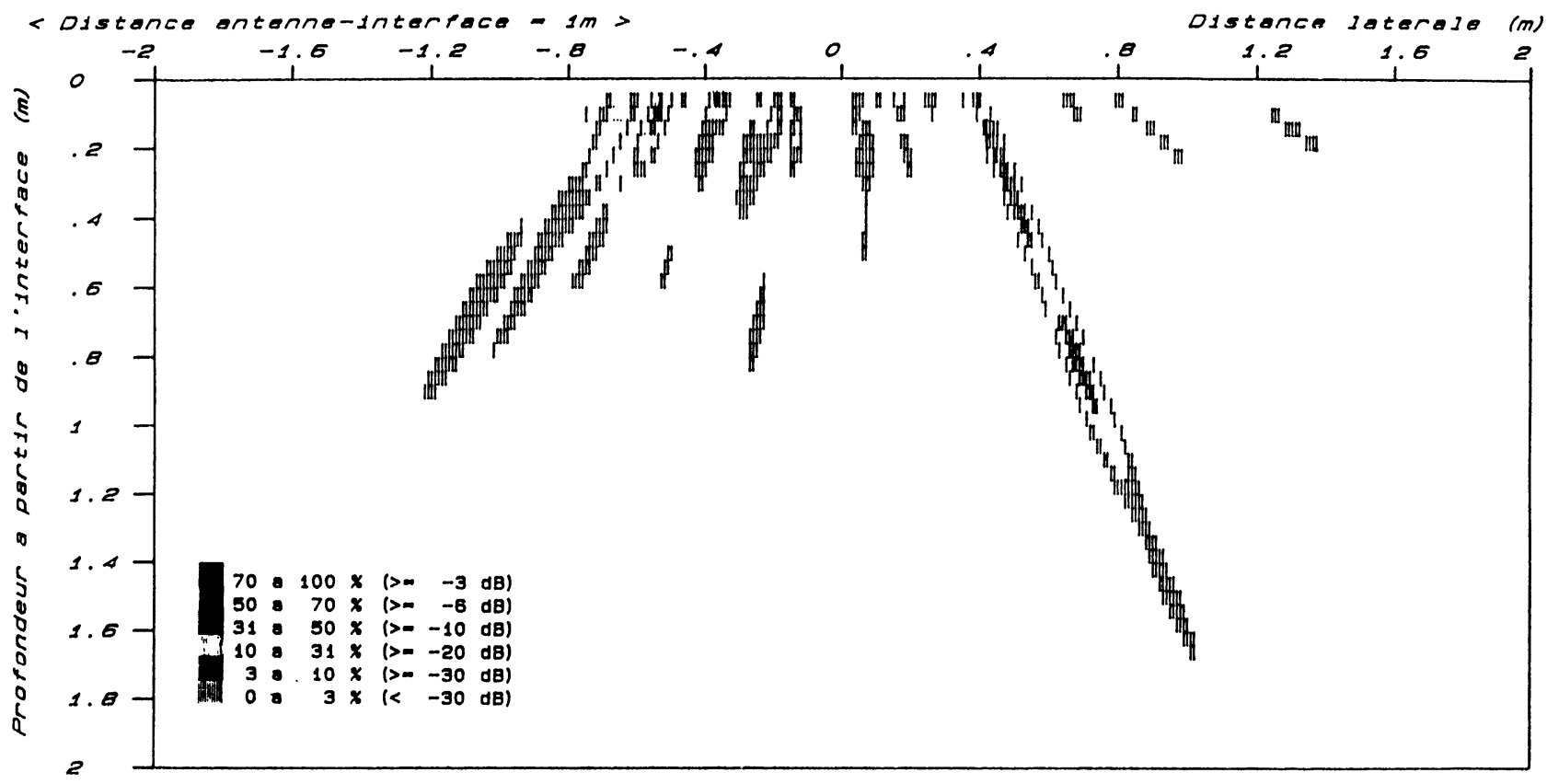

Fig. 6. - Modélisation du champ acoustique sous l'interface eau-sédiment en émission-réception (inclinaison $=20$ degrés).

[Transmission-reception acoustic field model through an interface (inclination $=20$ degrees).]

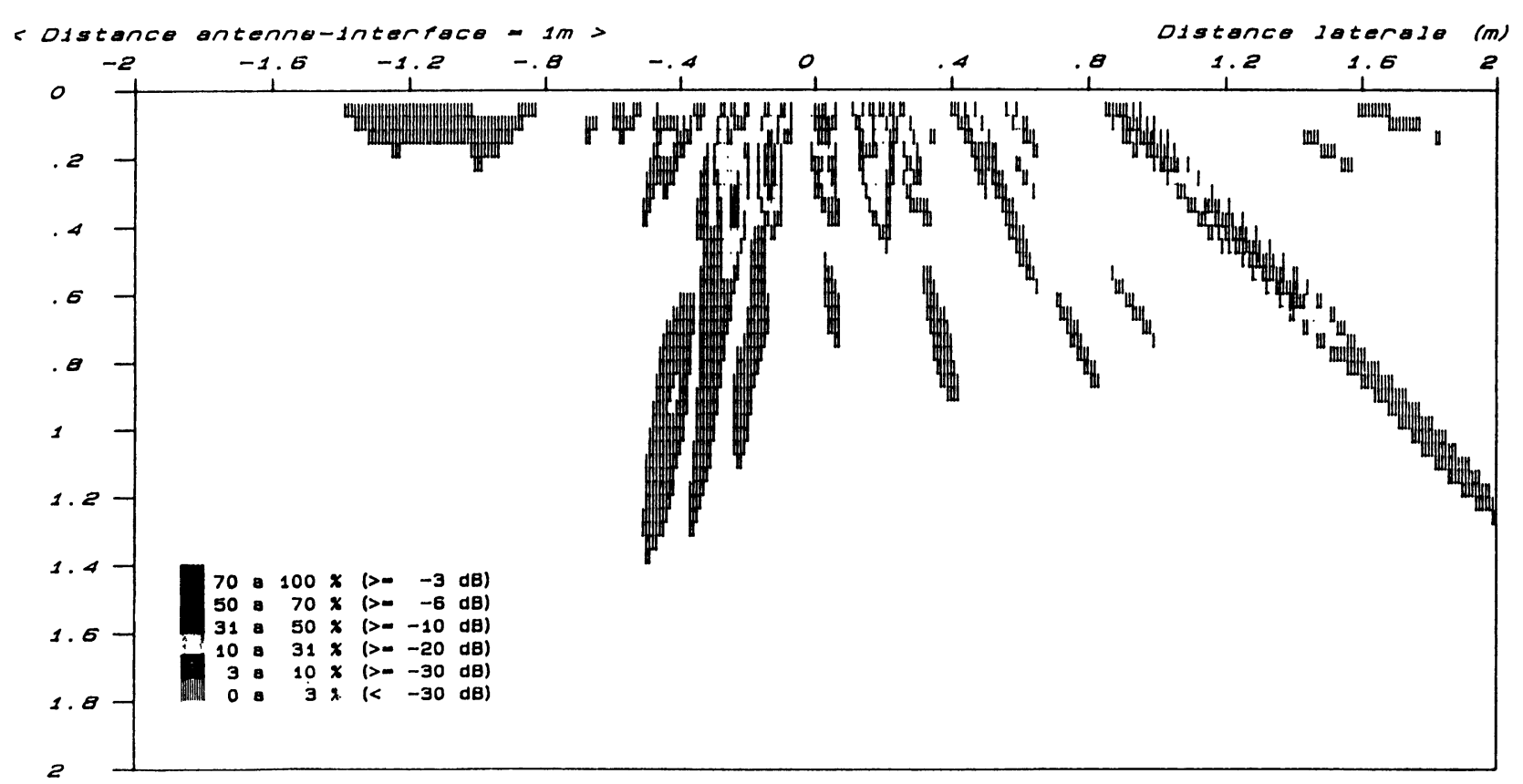

Fig. 7. - Modélisation du champ acoustique sous l'interface eau-sédiment en émission-réception (inclinaison $=40$ degrés).

[Transmission-reception acoustic field model through an interface (inclination $=40$ degrees).]

\section{Focalisation électronique par lignes à retard.}

La configuration annulaire de ce type d'antenne nécessite autant de lignes à retard que de couronnes utilisées.
5.1 RÉCEPTION. - Le seul procédé qui allie souplesse d'utilisation et simplicité est celui issu d'une technique hybride composée d'autant de portes analogiques (échantillonneur analogique) que de couronnes et d'un registre à décalage numérique [2]. 
Ce procédé rappelle la méthode hétérodyne dans laquelle le signal provenant de l'oscillateur local (issu du registre à décalage) à phase variable, est mélangé aux divers signaux des couronnes (Fig. 1).

a) Principe du mélangeur hétérodyne : Si les signaux en provenance respectivement de la couronne et de l'oscillateur local sont de la forme $V \sin \left(\omega t-\Gamma_{c}\right)$ et $V^{\prime} \sin \left(\omega^{\prime} t-\Phi_{c}\right)$, le signal en sortie du multiplieur (échantillonneur analogique) s'écrit :

$$
V_{1}=V V^{\prime} \sin \left(\omega t-\Gamma_{c}\right) \sin \left(\omega^{\prime} t-\Phi_{c}\right)
$$

soit :

$$
\begin{aligned}
V_{1}=V V^{\prime} / 2(\cos ( & \left.\left(\omega-\omega^{\prime}\right) t-\Gamma_{c}+\Phi_{c}\right)- \\
& \left.-\cos \left(\left(\omega+\omega^{\prime}\right) t-\Gamma_{c}-\Phi_{c}\right)\right) .
\end{aligned}
$$

Après filtrage centré sur le terme différence il ne reste que :

$$
V_{s c}=K V \cos \left(\left(\omega-\omega^{\prime}\right) t-\Gamma_{c}+\Phi_{c}\right)
$$

d'une façon générale si $\Phi_{c}=\alpha_{c r}-\Phi_{0}$, où $\Phi_{0}$ est un déphasage constant correspondant au déphasage maximum $\Phi_{c \max }$ de la couronne la plus externe, il vient :

$$
V_{\mathrm{sc}}=K V \cos \left(\left(\omega-\omega^{\prime}\right) t-\Phi_{0}\right)
$$

tous les signaux issus des couronnes sont alors en phase et leur traitement est optimum.

b) Réalisation de la loi de retard: On utilise un oscillateur de fréquence $F^{\prime}$ inférieure à la fréquence des ultrasons dont on fait varier la phase au moyen d'un registre à décalage à entrée série, sorties parallèles. Le signal $F^{\prime}$ injecté sur l'entrée série voit sa phase décalée de $m$ fois le retard élémentaire $\tau_{\mathrm{c}}=1 / F_{\Phi_{\mathrm{r}}}$ (où $m$ est le nombre de prises séparant la $1^{\text {rc }}$ sortie de la $m$-ième).

Le déphasage élémentaire est donné par: $\Phi_{\mathrm{c}}=\omega^{\prime} \tau_{\mathrm{e}}=2 \pi F^{\prime} / F_{\Phi_{\mathrm{r}}}$.

c) Cas des ondes sphériques (focalisation) : Dans le cas où l'on cherche à focaliser sur l'axe de l'antenne, le déphasage à introduire pour chaque couronne dépend du rayon de cette dernière.

D'une façon générale, et en prenant l'origine des phases comme centre de l'antenne et comme première prise du registre à décalage, il vient :

$$
\Phi_{c}=2 \pi \ell_{c} F / C_{\mathrm{e}}=m_{c \mathrm{r}} \Phi_{\mathrm{e}}=m_{c \mathrm{r}} 2 \pi F^{\prime} / F_{\Phi_{\mathrm{r}}}
$$

où

$\ell_{c} \approx a_{c}^{2} / 2 R_{0}$ correspond à la différence de trajet de

l'onde entre l'antenne plane et l'antenne courbe,

$a_{c}=$ rayon de la couronne $c$,

$F=$ fréquence de l'onde acoustique,

$R_{0}=$ distance de focalisation.

Ce qui permet de déterminer le nombre de prises séparant la sortie de l'oscillateur local relatif à une couronne et l'origine des phases, soit :

$$
m_{c \mathrm{r}}=F F_{\Phi \mathrm{r}} \ell_{c} / C F^{\prime} .
$$

Dans le cas d'un registre à 80 cellules de décalage et pour les paramètres suivants : $F=46 \mathrm{kHz}, \quad F^{\prime}=11,5 \mathrm{kHz}, \quad F_{\text {filtre }}=$ $F-F^{\prime}=34,5 \mathrm{kHz}, a_{2}=0,18 \mathrm{~m}, a_{3}=0,3 \mathrm{~m}$, $a_{4}=0,5 \mathrm{~m}$, il convient de sélectionner les prises :

$$
m_{2}=10, m_{3}=29, \quad m_{4}=80 .
$$

5.2 Emission. - Le traitement du signal se trouve simplifié car les différentes couronnes d'émission sont excitées par des trains d'onde décalés entre eux.

Ces signaux sont issus directement des prises d'un autre registre à décalage où le train d'onde à $46 \mathrm{kHz}$ provenant de la $m$-ième prise, est décalé de la quantité $m_{c \mathrm{c}} \tau_{\mathrm{e}}=m_{c \mathrm{e}} / F_{\Phi \mathrm{e}}$.

Ce qui pour la couronne de rayon $a_{c}$ doit correspondre au retard souhaité soit : $m_{c e}=F_{\Phi_{\mathrm{e}}} \ell_{c} / C_{\mathrm{e}}$.

En utilisant une loi de phases identique à celle de la réception, c'est-à-dire $m_{c r}=m_{c e}$ nous avons le tableau suivant avec $F_{\Phi_{\mathrm{r}}}=F_{\Phi \mathrm{e}} / 4$.

\begin{tabular}{|c|c|c|}
\hline Couronne & $\begin{array}{c}\text { Nb. de prises } \\
\text { en émission }\end{array}$ & $\begin{array}{c}\text { Nb. de prises } \\
\text { en réception }\end{array}$ \\
\hline$a(1)$ & 5 & \\
$a(2)$ & 10 & 10 \\
$a(3)$ & 29 & 29 \\
$a(4)$ & 80 & 80 \\
\hline
\end{tabular}

5.3 Remarques. - Il est à noter toutefois que ce modèle de «loi de phases" pour des raisons de divergence de l'onde acoustique déjà entrevues dans le chapitre 3.1, ne produit pas l'effet escompté c'està-dire la présence d'un maximum de pression à l'endroit désiré. La focalisation avec ce modèle s'opère avant la distance théorique calculée.

Ce léger inconvénient est vite contourné en ajustant les fréquences $F_{\Phi_{\mathrm{e}}}$ et $F_{\Phi_{\mathrm{r}}}$ des registres à décalage (en augmentant celles-ci) pour focaliser au point sou ait , ce qui revient a s a 1gner sur a « 01 de phases » obtenue par calcul numérique.

Ce type de lignes à retard permet, par la variation des fréquences de décalage $F_{\Phi_{\mathrm{r}}}$ et $F_{\Phi_{\mathrm{e}}}$, c'est-à-dire de la distance de focalisation, d'effectuer un « zoom électronique ».

D'autre part, la «loi de phases » peut être modifiée par action sur les prises du registre à décalage.

Le balayage latéral du faisceau acoustique pourrait utiliser le même principe avec toutefois la nécessité d'introduire autant de lignes à retard que de capteurs. 


\section{Résultats expérimentaux.}

6.1 InSTRUMENTATION. - L'expérimentation de l'antenne à focalisation a été effectuée sur un site d'essais comprenant une piscine en eau de $6 \times 3 \times 2 \mathrm{~m}$ et un bassin à sédiments de $3 \times 3 \times 4,5 \mathrm{~m}$.

Dans le cas des relevés de réponses acoustiques et d'imagerie dans l'eau, l'antenne est immergée à $1,5 \mathrm{~m}$ du bord (afin d'éviter les échos parasites arrières) avec sa face active dirigée suivant la plus grande dimension de la piscine, permettant ainsi des mesures jusqu'à $3 \mathrm{~m}$ de distance.

Le réseau de capteurs pivote autour d'un axe radial commandé par le micro-ordinateur via le moteur pas-à-pas. La résolution angulaire qu'apporte un tel système est de $0,5^{\circ}$.

Les différents relevés cartographiques de pressions ont nécessité le balayage angulaire du faisceau acoustique conjointement au déplacement longitudinal d'un transducteur (tantôt émetteur, tantôt récepteur, puis faisant office de cible réfléchissante).

L'information reçue par ce transducteur ou par l'antenne, est traitée par l'électronique du système puis visualisée sur l'écran du micro-ordinateur et enfin sauvegardée dans des fichiers. Un traitement ultérieur, notamment par conversion des coordon- nées polaires en coordonnées cartésiennes, rend possible la comparaison des cartes obtenues par modélisation et par expérimentation.

En ce qui concerne les essais d'imagerie dans le sédiment, l'antenne supportée par un bâti articulé peut pivoter selon 2 axes radiaux orthogonaux.

L'ensemble est placé dans l'eau à $0,7 \mathrm{~m}$ au-dessus de l'interface eau-sédiment, pointé en direction des cibles enfouies sous le sédiment.

6.2 RELEVÉs DE RÉPONSES ACOUSTIQUES. L'antenne fonctionne en réception. Le relevé de la carte isobare en 3 dimensions (Fig. 8) correspond à l'échantillonnage du diagramme de rayonnement du réseau de transducteurs pour une distance de focalisation de $2 \mathrm{~m}$. Cette représentation est à rapprocher du modèle théorique (Fig. 3). La dissymétrie autour de l'axe de l'antenne des lobes de rayonnement est inhérente à la non-reproductibilité des capteurs. De plus, l'influence de ces lobes secondaires semble être moindre que ne le laisse supposer le modèle théorique. Ce phénomène, déjà observé par d'autres auteurs [4-5] provient de la non-ponctualité des transducteurs. Nous avons également relevé le champ acoustique du réseau en émission-réception (Fig. 9). La cartographie obtenue, que l'on peut comparer au modèle théorique (Fig. 4) fait apparaî-

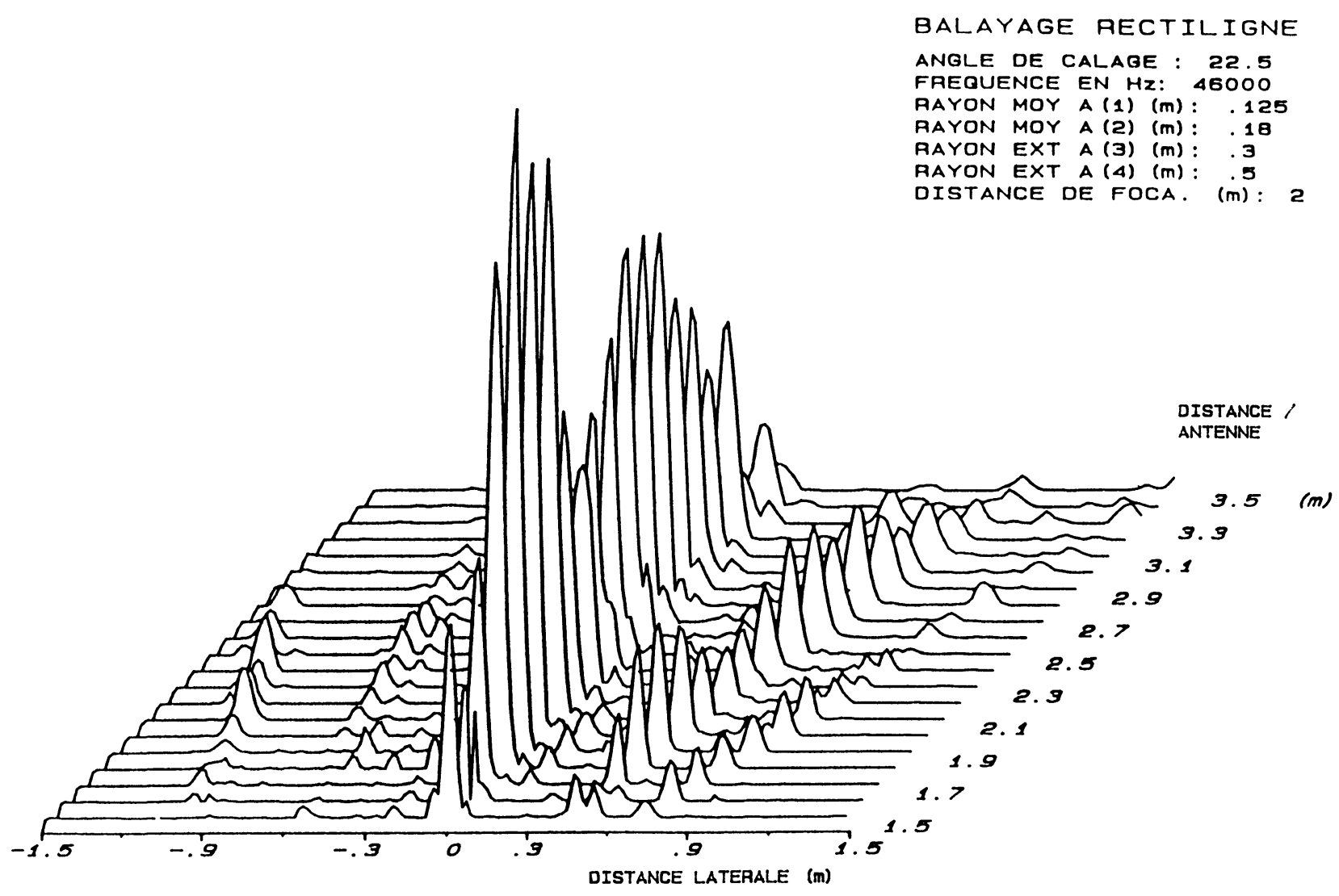

Fig. 8. - Relevé in situ du champ acoustique dans l'eau en réception.

[In situ received underwater acoustic field.] 
BALAYAGE RECTILIGNE

aNGLE de calage: 22.5 FPEQUENCE EN Hz: i 48000 PAYON MOY $A(2)(\mathrm{m}): .18$ RAYON EXT A (3) $(\mathrm{m}): .3$ RAYON EXT A (4) (m): .5 DISTANCE DE FOCA. $(m): 2$

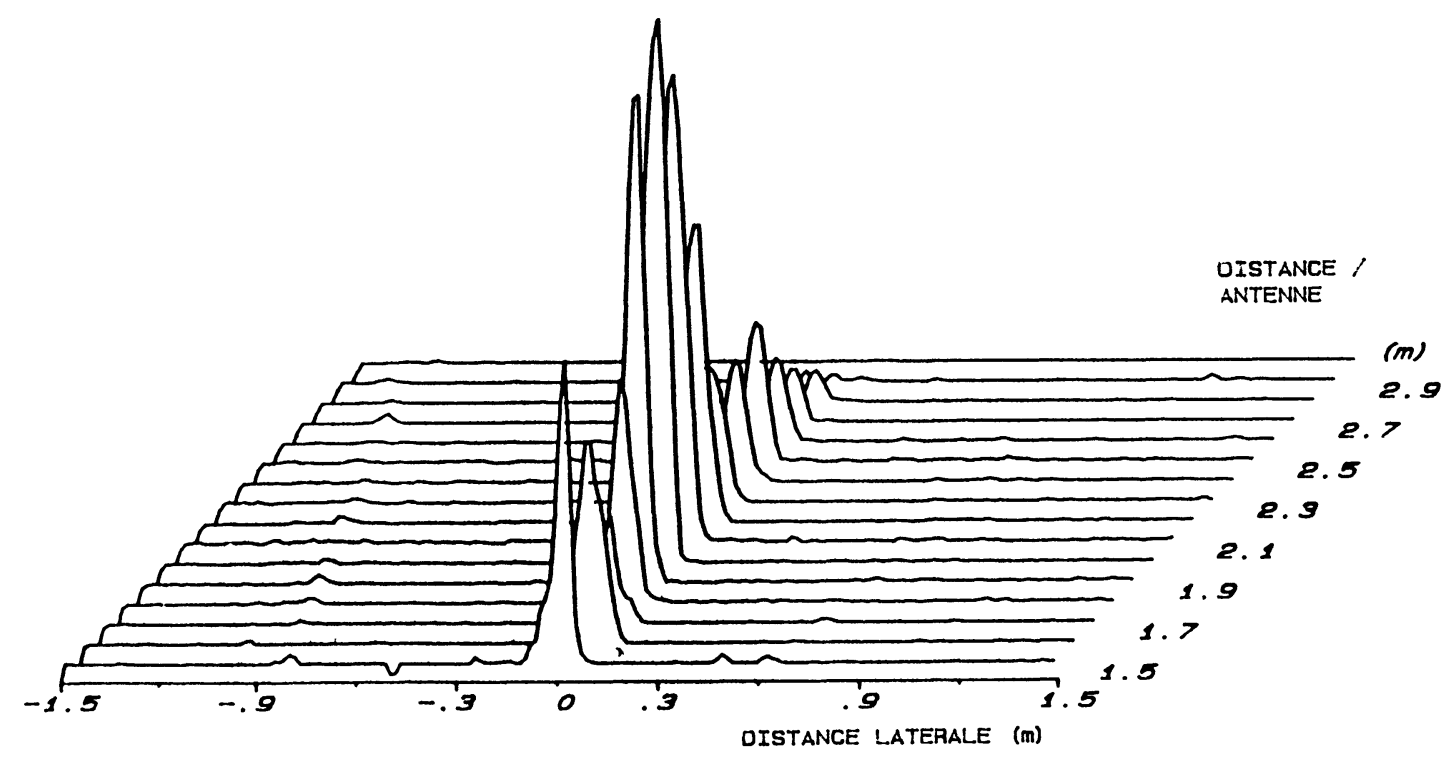

Fig. 9. - Relevé in situ du champ acoustique dans l'eau en émission-réception.

[In situ transmission-reception underwater acoustic field.]

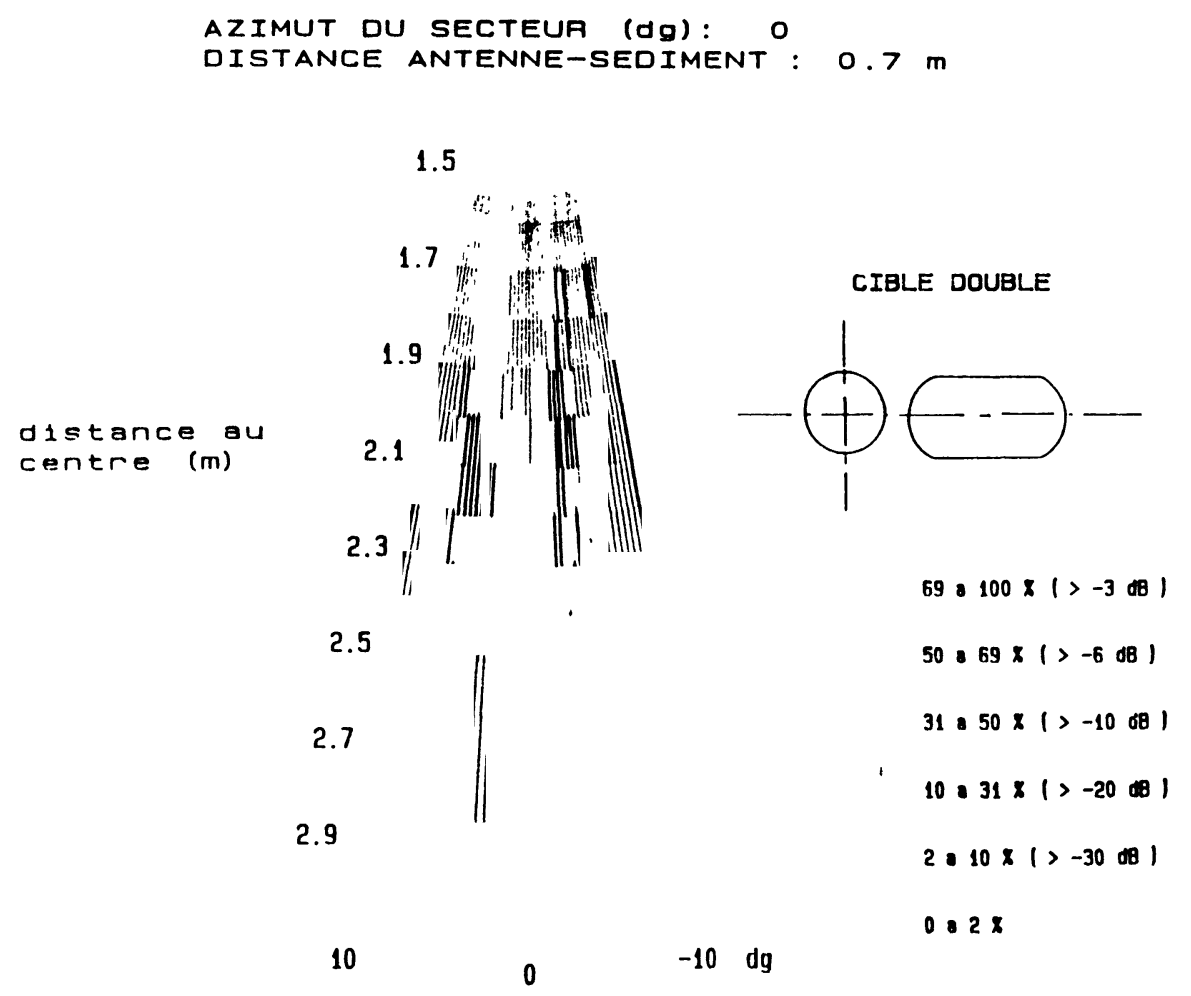

Fig. 10. - Pouvoir séparateur dans le cas d'imagerie dans l'eau.

[Underwater Image resolution.] 
tre un pic central à la cote $2 \mathrm{~m}\left(F_{\Phi_{\mathrm{r}}}=400 \mathrm{kHz}\right.$, $\left.F_{\Phi \mathrm{c}}=1,9 \mathrm{MHz}\right)$ de dimensions axiale et radiale à $-3 \mathrm{~dB}$ respectivement égales à $30 \mathrm{~cm}$ et $4 \mathrm{~cm}$.

On remarquera un niveau très faible des lobes secondaires, qui au voisinage du point de focalisation est inférieur de $-35 \mathrm{~dB}$ par rapport au pic central. Ce meilleur résultat est à attribuer au caractère non ponctuel des transducteurs élémentaires déjà évoqués que ne prend pas en compte la théorie.

6.3 IMAGERIE. - La construction d'images à partir du champ rétrodiffusé par plusieurs types de cibles permet d'appréhender le pouvoir séparateur de l'antenne.

Les premiers essais d'imagerie effectués en bassin rempli d'eau ont permis d'apprécier la résolution axiale et radiale de l'antenne. Pour cela, nous avons balayé un secteur angulaire de $\pm 10^{\circ}$ autour d'une cible métallique double composée d'un disque (diamètre $=130 \mathrm{~mm}$ ) et d'un ovale (longueur $=150 \mathrm{~mm}$ ) distants de $5 \mathrm{~cm}$. Par déplacement angulaire et axial (zoom électronique) de la tache focale nous avons reconstitué la position des cibles (Fig. 10). Lors de cette expérience, l'échantillonnage spatial qu'aurait pu apporter la fenêtre de détection couplée à la distance focale, était désactivé.

Le même type d'essais, sur des cibles enfouies dans un sédiment artificiel (kaolinite) s'est heurté aux problèmes de réflexions multiples par confinement à l'intérieur du bassin d'essais. Les prochains essais en mer ne devraient pas présenter cet inconvénient.

Tableau I. - Résultats théoriques et expérimentaux des dorsales en émission-réception.

[Theoricals and experiments results of dorsals (transmission-reception).]

\begin{tabular}{|c|c|c|c|c|}
\hline $\begin{array}{c}\text { Fréquence de } \\
\text { focalisation } \\
\text { en émission } \\
F(\mathrm{MHz})\end{array}$ & $\begin{array}{c}\text { Fréquence de } \\
\text { focalisation } \\
\text { en réception } \\
F(\mathrm{kHz})\end{array}$ & $\begin{array}{c}\text { Distance de } \\
\text { focalisation } \\
R O(\mathrm{~m})\end{array}$ & \multicolumn{2}{|c|}{$\begin{array}{c}\text { Longueur de la tache focale } \\
\text { à }-3 \mathrm{~dB}(\mathrm{~cm})\end{array}$} \\
\hline 1,14 & 300 & 1,30 & pratique & théorique \\
1,18 & 350 & 1,70 & 11 & - \\
1,90 & 400 & 1,97 & 17 & - \\
2,30 & 450 & 2,27 & 30 & - \\
2,70 & 500 & 2,55 & 33 & - \\
2,95 & 550 & 2,78 & 50 & - \\
3,25 & 600 & 2,95 & 56 & 72 \\
3,50 & 650 & 3 & 66 & \\
\hline
\end{tabular}

Tableau II. - Résultats théoriques et expérimentaux des diagrammes de rayonnement en émission-réception. [Theoricals and experiments results of responses pattern (transmission-reception).]

\begin{tabular}{|c|c|c|c|c|c|c|}
\hline \multirow{2}{*}{$\begin{array}{c}\text { Fréquence de } \\
\text { focalisation } \\
\text { en émission } \\
F(\mathrm{MHz})\end{array}$} & \multirow{2}{*}{$\begin{array}{c}\begin{array}{c}\text { Fréquence de } \\
\text { focalisation } \\
\text { en réception }\end{array} \\
F(\mathrm{kHz})\end{array}$} & \multirow{2}{*}{$\begin{array}{c}\begin{array}{c}\text { Distance de } \\
\text { focalisation }\end{array} \\
R O(\mathrm{~m})\end{array}$} & \multicolumn{2}{|c|}{ Pratique } & \multicolumn{2}{|c|}{ Théorique } \\
\hline & & & $\begin{array}{c}\text { angle } \\
\text { d'ouverture } \\
\text { à }-3 \mathrm{~dB} \\
\theta \text { (degrés) }\end{array}$ & $\begin{array}{l}\text { niveau max } \\
\text { des lobes } \\
\text { secondaires } \\
\text { (dB) }\end{array}$ & $\theta$ (degrés) & (dB) \\
\hline 1,14 & 300 & 1,30 & 1,30 & $-35,00$ & - & - \\
\hline 1,18 & 350 & 1,70 & 1,30 & $-32,00$ & - & - \\
\hline 1,90 & 400 & 2,00 & 1,30 & $-35,00$ & 1 & -21 \\
\hline 2,70 & 500 & 2,55 & 1,20 & $-35,50$ & - & - \\
\hline 3,25 & 600 & 2,85 & 1,20 & $-26,80$ & - & - \\
\hline 3,70 & 700 & 3,10 & 0,90 & $-22,50$ & 1 & -17 \\
\hline
\end{tabular}




\section{Conclusion.}

Nous avons expérimenté en bassin une méthode de reconstruction d'images à partir d'un réseau de capteurs à focalisation. Les caractéristiques acoustiques de l'antenne en émission-réception sont meilleures que celles prévues par la théorie (voir Tabs. III). Ceci essentiellement à cause de la directivité des transducteurs qui n'est pas pris en compte par le modèle théorique.

L'étude théorique de focalisation dans les sédiments menée conjointement avec des essais d'imagerie dans l'eau permet d'envisager la détection d'objets enfouis sous quelques mètres de sédiments.

Le principe de la focalisation électronique sera étendu au balayage latéral du faisceau accroissant ainsi la cadence d'images.

\section{Bibliographie}

[1] Shibata S., Koda T., Yamaga J., C-lide ultrasonic imaging by and electronically scanned coaxial circular spherical receiving array, Ultrasonics 6568 (1978).

[2] Salvini G., Calaora A., Gazahnes C., Imageries en temps réel par traitement d'antenne hybride, Trait. sign. 4 (1986) 329-334.

[3] Fink M., L'imagerie ultrasonore, Revue Phys. Appl. 18 (1983) 527-558.

[4] Delannoy B., Reconstruction d'images acoustiques par échantillonnage et correction de phase, Thèse de Doctorat d'Etat, Université de Valenciennes, 152 p., juin 1979.

[5] Salvini G., Calaora A., Gazahnes C., Traitement d'antenne hybride, Neuvième colloque sur le traitement du signal et ses applications (1983) pp. $811-815$.
[6] Bresson J., BARriol R., LONGUemard J. P., Antenne acoustique multi-éléments à focalisation, Modélisation du champ acoustique, Revue Phys. Appl. 22 (1987) 1177-1184.

[7] Salvini G., Calaora A., Calcul et réalisation d'antennes à éléments multiples, Rev. Cethedec NS80.1 (1980) 261-273.

[8] Hamilton E. L., Sound velocity density relations in sea floor sediments and rocks, J. Acoust. Soc. Am. 63 (2) (1974).

[9] Frohly J., Etude de la propagation des ondes ultrasonores dans les milieux hétérogènes et application de l'imagerie en mode $B$ au contrôle non destructif, Thèse d'Etat présentée à l'Université de Valenciennes, 149 p., avril 1983. 